\title{
Safe and successful treatment with afatinib in three postoperative non-small cell lung cancer patients with recurrences following gefitinib/erlotinib-induced hepatotoxicity
}

\author{
Hiroaki Toba, Shoji Sakiyama, Hiromitsu Takizawa, and Akira Tangoku \\ Department of Thoracic and Endocrine Surgery and Oncology, Institute of Health Biosciences, University of Tokushima Graduate School
}

\begin{abstract}
Background : Gefitinib and/or erlotinib-induced hepatotoxicity sometimes lead to treatment failure in EGFR mutation - positive patients with non-small cell lung cancer (NSCLC), even though the therapeutic effect is evident. Cases : Here, we report three postoperative NSCLC patients with recurrences who experienced severe hepatotoxicity while receiving gefitinib and/or erlotinib treatment but could be safely switched to afatinib treatment. Conclusion : Afatinib could be a well-tolerated EGFR-TKI that could be chosen for its relatively low hepatotoxicity, which is attributable to its having a different metabolic mechanism compared to other EGFRTKIs. J. Med. Invest. 63 : 149 - 151, February, 2016
\end{abstract}

Keywords : afatinib, gefitinib/erlotinib-induced hepatotoxicity, postoperative recurrence, non-small cell lung cancer

\section{INTRODUCTION}

Gefitinib and/or erlotinib-induced hepatotoxicity sometimes lead to treatment failure in EGFR mutation-positive patients with non-small cell lung cancer (NSCLC), even though the therapeutic effect is evident. Afatinib is a second-generation EGFR-TKI with a metabolism different from those of gefitinib and erlotinib, although severe transaminase elevation of grade $\geq 3$ occurs in $0.4 \%$ of afatinib-treated patients (1). Here, we report three postoperative NSCLC patients with recurrences who experienced severe hepatotoxicity while receiving gefitinib and/or erlotinib treatment but could be safely switched to afatinib treatment.

\section{Case 1}

A 73-year-old nonsmoking female received left upper lobectomy for adenocarcinoma of the lung (pT2aN0M0, Stage IB, EGFR : exon 21 L858R mutation) (Case 1 in Table 1). Bilateral adrenal metastases occurred at 8 months after operation (Figure 1A). Gefitinib treatment was initiated. After 8 weeks, her serum AST and ALT had increased markedly to $253 \mathrm{U} / \mathrm{L}$ and $316 \mathrm{U} / \mathrm{L}$, respectively (grade 3 toxicity according to the Common Terminology Criteria for Adverse Events (CTCAE, version 4.0) (Figure 1B) although computed tomography $(\mathrm{CT})$ revealed tumor size reductions. Next, she received erlotinib treatment. After 6 weeks, her serum ASL and ALT levels had increased markedly to $615 \mathrm{U} / \mathrm{L}$ and $442 \mathrm{U} / \mathrm{L}$ (grade 3) again (Figure 1B). After the regrowth of recurrent sites, afatinib treatment was finally commenced as a fourth-line treatment at a daily dose of $20 \mathrm{mg}$ at 8 months after recurrence. After 7 months of afatinib administration, CT demonstrated that the tumor sizes were reduced without an elevation of transaminase (Figure $1 \mathrm{~B}$ and $1 \mathrm{C}$ ).

Received for publication September 18, 2015 ; accepted January 12, 2016. Address correspondence and reprint requests to Hiroaki Toba, Thoracic and Endocrine Surgery and Oncology, Institute of Health Biosciences, University of Tokushima Graduate School, 18-15 Kuramoto-cho 3, Tokushima 770-8503, Japan and Fax : +81-88-633-9031.
Case 2

A 63-year-old nonsmoking male received right middle lobectomy for adenocarcinoma of the lung (pT2aN1M0, Stage IIA, EGFR : exon 21 L858R mutation) (Case 2 in Table 1). Multiple plural disseminations occurred at 73 months after operation. Gefitinib treatment was initiated. After 4 weeks, his serum AST and ALT had increased markedly to $267 \mathrm{U} / \mathrm{L}$ and $414 \mathrm{U} / \mathrm{L}$ (grade 3). Afatinib treatment was commenced as a second-line treatment at a daily dose of $30 \mathrm{mg}$ at 4 months after recurrence. After 7 months of afatinib administration, CT revealed tumor size reductions without an elevation of transaminase.

Case 3

A 63-year-old nonsmoking female received right upper lobectomy for adenocarcinoma of the lung (pT2aN0M0, Stage IB, EGFR: exon 19 deletion) (Case 3 in Table 1). Multiple plural disseminations, and pulmonary and mediastinal lymph node metastases occurred with elevated carcinoembryonic antigen (CEA) at 28 months after operation. Gefitinib treatment was initiated. After 8 weeks, her serum AST and ALT had markedly increased to 397 $\mathrm{U} / \mathrm{L}$ and $687 \mathrm{U} / \mathrm{L}$ (grade 3) although tumor size reductions were evident. After regrowth of the recurrent sites, afatinib treatment was finally commenced at a daily dose of $20 \mathrm{mg}$ as a fifth-line treatment at 23 months after recurrence. After 7 months of afatinib administration, the serum CEA levels showed a marked decrease and CT revealed tumor size reductions without an elevation of transaminase.

\section{DISCUSSION}

This is the first report to demonstrate that afatinib treatment could be safely and successfully performed following geftinib/erlotinibinduced hepatotoxicity in postoperative NSCLC patients with recurrences.

EGFR-TKIs are well known to be key therapeutic drugs for EGFR mutation-positive NSCLC patients. However, despite their evident therapeutic efficacy, they sometimes have to be discontinued due 
Table 1. Charactetrstics of patients

\begin{tabular}{|c|c|c|c|}
\hline & Case 1 & Case 2 & Case 3 \\
\hline Age (year)/Sex & $73 / \mathrm{F}$ & $63 / \mathrm{M}$ & $63 / \mathrm{F}$ \\
\hline PS & 1 & 0 & 0 \\
\hline Smoking & Never & Never & Never \\
\hline Histology & Ad & Ad & Ad \\
\hline Operation & Left upper lobectomy & Right middle lobectomy & Right upper lobectomy \\
\hline Pathological stage & IB & IIA & IB \\
\hline EGFR-mutation & Exon 21 L858R & Exon 21 L858R & Exon 19 delation \\
\hline Recurrentsite & Bilateral adrenal glands & Pleural dissemination & $\begin{array}{l}\text { Pleural dissemination } \\
\text { Lung } \\
\text { Mediastinal lymoh node }\end{array}$ \\
\hline D.F.I. (months) & 8 & 73 & 28 \\
\hline Previous treatments & $\begin{array}{l}\text { 1st. Gefitinib } \\
\text { 2nd. Erlotinib } \\
\text { 3rd. CBDCA/PEM }\end{array}$ & 1st. Gefitinib & $\begin{array}{l}\text { 1st. CBDCA/PEM } \\
\text { 2nd. Gefitinib } \\
\text { 3rd. S-1 } \\
\text { 4th. DOC }\end{array}$ \\
\hline Hepatotoxicity (grade) & 3 & 3 & 3 \\
\hline Dose of afatinib (mg) & 20 & 30 & 20 \\
\hline $\begin{array}{l}\text { Duration of afatinib treatment } \\
\text { (months) }\end{array}$ & 4 & 4 & 4 \\
\hline Response & $\mathrm{PR}$ & $\mathrm{PR}$ & $\mathrm{PR}$ \\
\hline Adverse events & $\begin{array}{l}\text { Paronychia (grade 2) } \\
\text { Diarrhea (grade 1) } \\
\text { Taste disturbance (grade 1) }\end{array}$ & $\begin{array}{l}\text { Skin rush (grade 2) } \\
\text { Diarrhea (grade 1) }\end{array}$ & Paronychia (grade 1 ) \\
\hline
\end{tabular}

Ad : adenocarcinoma, PS : performance status, D.F.I. : disease free interval, CBDCA : carboplatin, PEM : pemetrexed, DOC : docetaxel, PR : partial response
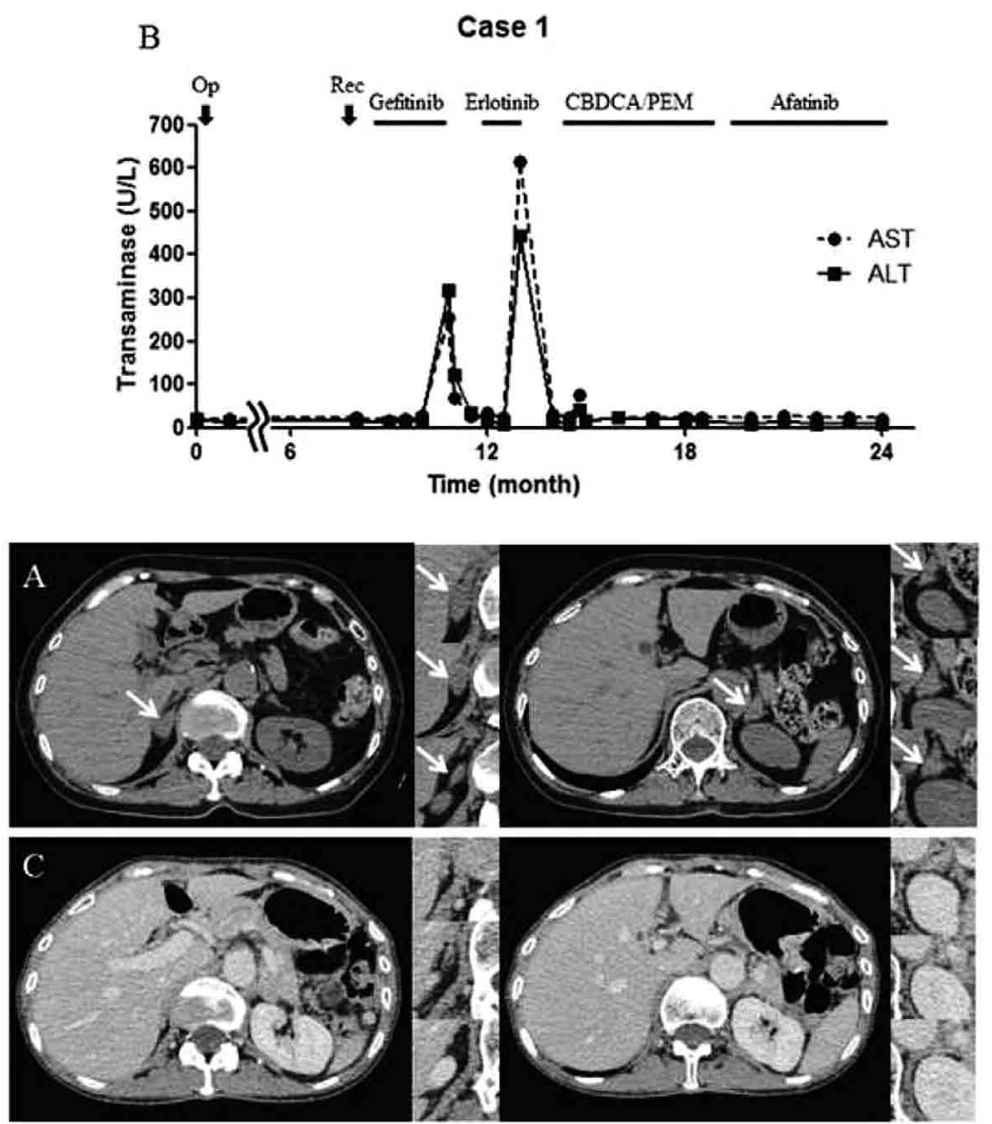

Figure 1. Clinical course in Case 1. A : Computed tomography (CT) revealed enlargement of the bilateral adrenal glands (arrows) at 8 months after operation. B : Changes in alanine aminotransferase (ALT) and aspartate aminotransferase (AST) during gefitinib, erlotinib and afatinib treatment. $\mathrm{C}: \mathrm{CT}$ revealed the size reduction of recurrent sites after afatinib treatment.

Op : operation, Rec : recurrence, CBDCA/PEM : Carboplatin/Pemetrexed. 
to hepatotoxicity or other adverse events (AEs). Thus one study reported a severe transaminase elevation of grade $\geq 3$ in $23.6 \%$ of gefitinib-treated Japanese patients (the WJTOG3405 study) (2). Afatinib is a second-generation EGFR-TKI and an irreversible inhibitor of all ERBB family receptor tyrosine kinases, and has already shown favorable results for EGFR mutation-positive NSCLC patients in the LUX-Lung 3 study (1). It also induces grade $\geq 3$ hepatotoxicity in $0.4 \%$ of patients (1). However, it has a different metabolic mechanism than gefitinib and erlotinib. Gefitinib and erlotinib are metabolized in the liver by cytochrome P450 enzymes (CYP) such as CYP3A4, with more than $80 \%$ of the administered dose being found in feces (3). On the other hand, Stopfer et al. (4) indicated that the metabolism of afatinib is negligible because it exhibits high plasma protein binding of $94.6 \%$ in healthy volunteers, and only a small fraction of the total plasma concentration is directly exposed to hepatic metabolism and excretion. This difference has been considered the reason why afatinib treatment exhibits much lower hepatotoxicity compared to gefitinib/erlotinib treatment.

In case 1 , both gefitinib and erlotinib were administered, and grade 3 hepatotoxicity occurred even though the therapeutic effects were evident. Conventional chemotherapy would be chosen before afatinib in such a case. However, in this case we judged that an EGFR-TKI would be effective, and indeed, administration of afatinib has so far provided a major therapeutic benefit without hepatotoxicity. On the other hand, in cases 2 and 3, afatinib treatment was directly chosen after the failure of gefitinib treatment, even though erlotinib might not cause severe hepatotoxicity, because 1) the incidence of hepatotoxicity was thought to be much less, and 2) an EGFR-TKI was expected to have an greater therapeutic effect. Takimoto et al. (5) reported that polymorphisms of the CYP2D6 gene are associated with gefitinib- and/or erlotinib-induced hepatotoxicity, and thus analysis of these polymorphisms might lead to the choice of an appropriate EGFR-TKI, and a corresponding reduction in drug-induced hepatotoxicity.

Kato et al. (6) analyzed just Japanese patients in the LUX-Lung 3 trial. Consequently, $75.9 \%$ Japanese patients had dose reductions to $30 \mathrm{mg}(33.3 \%)$ and $20 \mathrm{mg}(42.6 \%)$ due to AEs. However,progression-free survival was significant longer with afatinib than cisplatin/pemeterxed, indicating that the lower dose is also acceptable in Japanese patients in terms of its efficacy. That is the reason why we used the lower doses in these 3 patients.

At the present time, afatinib should be used as a second-line EGFR-TKI. However, in the future, afatinib might potentially be chosen as a first-line EGFR-TKI if its effect can be shown to be as good as or better than gefitinib and erlotinib in LUX-Lung 7 and 8 studies.

\section{CONCLUSION}

Our case report indicated that afatinib could be a well-tolerated EGFR-TKI that could be chosen for its relatively low hepatotoxicity, which is attributable to its having a different metabolic mechanism compared to other EGFR-TKIs.

\section{CONFLICTS OF INTEREST}

None declared.

\section{REFERENCES}

1. Sequist LV, Yang JC, Yamamoto N, O’Byrne K, Hirsh V, Mok T, Geater SL, Orlov S, Tsai C, Boyer M, Su W, Bennouna J, Kato T, Gorbunova V, Lee KH, Shah R, Massey D, Zazulina V, Shahidi M, Schuler M : Phase III study of afatinib or cisplatin plus pemetrexed in patients with metastatic lung adenocarcinoma with EGFR mutations. J Clin Oncol 31 : 3327-34, 2013

2. Mitsudomi T, Morita S, Yatabe Y, Negoro S, Okamoto I, Tsurutani J, Seto T, Satouchi M, Tada H, Hirashima T, Asami K, Katakami N, Takada M, Yoshioka H, Shibata K, Kudoh S, Shimizu E, Saito H, Toyooka S, Nakagawa K, Fukuoka M : Gefitinib versus cisplatin plus docetaxel in patients with nonsmall-cell lung cancer harbouring mutations of the epidermal growth factor receptor (WJTOG3405) : an open label, randomised phase 3 trial. Lancet Oncol 11 : 121-8, 2010

3. Ling J, Johnson KA, Miao Z, Rakhit A, Pantze MP, Hamilton M, Lum BL, Prakash C : Metabolism and excretion of erlotinib, a small molecule inhibitor of epidermal growth factor receptor tyrosine kinase, in healthy male volunteers. Drug Metab Dispos 34 : 420-6, 2006

4. Stopfer P, Marzin K, Narjes H, Gansser D, Shahidi M, Uttereuther-Fischer M, Ebner T: Afatinib pharmacokinetics and metabolism after oral administration to healthy male volunteers. Cancer Chemother Pharmacol 69 : 1051-61, 2012

5. Takimoto T, Kijima T, Otani Y, Nonen S, Namba Y, Mori M, Yokota S, Minami S, Komuta K, Uchida J, Imamura F, Furukawa M, Tsuruta N, Fujio Y, Azuma J, Tachibana I, Kumanogoh A : Polymorphisms of CYP2D6 Gene and Gefitinib-Induced Hepatotoxicity. Clin Lung Cancer 14 : 502-7, 2013

6. Kato T, Yoshioka H, Okamoto I, Yokoyama A, Hida T, Seto T, Kiura K, Massey D, Seki Y, Yamamoto N : Afatinib versus cisplatin plus pemetrexed in Japanese patients with advanced non-small cell lung cancer harboring activating EGFR mutations : Subgroup analysis of LUX-Lung 3. Cancer Sci 106 : 1202-1211, 2015 